Supporting Information for

\title{
Palladium-Catalyzed Cross-Coupling of Pyrrole Anions with Aryl Chlorides, Bromides and Iodides
}

\author{
Ryan D. Rieth, Neal P. Mankad, Elisa Calimano, and Joseph P. Sadighi*
}

\section{Experimental Section}

General considerations: Unless stated otherwise, all synthetic experimentation was carried out using Schlenk techniques under an argon atmosphere, or in an Innovative Technologies glovebox under a nitrogen atmosphere. Reactions were carried out in flame-dried glassware cooled under vacuum. Anhydrous hexanes and tetrahydrofuran (THF), inhibitor-free, were purchased from Aldrich in 18-L Pure-Pac ${ }^{\mathrm{TM}}$ solvent kegs, and sparged vigorously with argon for 40 minutes prior to first use. Hexanes were further purified by passage through one column of neutral alumina and one column of copper(II) oxide; THF, by passage through two columns of neutral alumina and one column of activated $4 \AA$ molecular sieves. 1,4-Dioxane, anhydrous, was purchased from Aldrich in Sure-Seal ${ }^{\mathrm{TM}}$ bottles and used as received.

Iodobenzene, 3-bromoquinoline, 2-chloroanisole, and 1-bromo-4-tert-butylbenzene were purchased from Aldrich and were either filtered through alumina (EM Science, 80-325 mesh) or stored over $3 \AA$ molecular sieves prior to use. Palladium acetate, 4-bromotoluene, and 2,4-dimethylpyrrole were purchased from Aldrich and used as received. Sodium hydride (60\% dispersion in mineral oil) was purchased from Aldrich. For in situ deprotonation of pyrroles (see General Procedure B, below) it was used as received. For all other reactions, it was washed free of mineral oil with anhydrous hexanes under inert atmosphere, and dried in vacuo. Pyrrole was purchased from Aldrich, stored over $3 \AA$ molecular sieves, and sparged for 20 min with argon prior to each use. 2-Bromomesitylene was purchased from Acros Chemical company and filtered through alumina (EM Science, 80-325 mesh) prior to use. 3,5-Dimethoxychlorobenzene was purchased from Acros Chemical company and was used 
as received. 1-Bromo-2,4,6-triisopropylbenzene was purchased from Avocado Chemical company and filtered through alumina (EM Science, 80-325 mesh) prior to use. 4-Bromo- $N, N$-dimethylaniline was purchased from Avocado Chemical company and used as received. 1-Chloro-4-(n-butyl)benzene was purchased from Lancaster Chemical company and filtered through alumina (EM Science, 80-325 mesh) prior to use. 3,5-Bis(trifluoromethyl)bromobenzene was purchased from Oakwood Products and was filtered through alumina (EM Science, 80-325 mesh) prior to use. Anhydrous zinc chloride beads were purchased from Alfa Aesar and used as received. 3-Methylpyrrole was purchased from TCI and used as received. 2-(Di-tert-butylphosphino)-biphenyl, 2-(dicyclohexylphosphino)biphenyl, 2-(dicyclohexylphosphino)-2'-methylbiphenyl, 2-(di-tert-butylphosphino)-2'-( $N, N$-dimethylamino)biphenyl, 2-dicyclohexylphosphino-2'-(N,N-dimethylamino)biphenyl, and 2-(dicyclohexylphosphino)-2',6'-dimethoxybiphenyl were purchased from Strem Chemical company and used as received. Ethyl acetate (HPLC grade) and diethyl ether (Reagent ACS grade) were used as received from Mallinckrodt. Hexanes for chromatography (HPLC grade) were used as received from Burdick \& Jackson. Silica gel (230-400 mesh) was used as received from EMD Chemicals, Inc. TLC plates (Silica Gel 60, $\mathrm{F}_{254}$ ) were purchased from EM Science.

IR spectra were recorded on a Perkin Elmer 2000 series FT-IR as $\mathrm{KBr}$ pellets. $\mathrm{CDCl}_{3}$ and acetone- $d_{6}$ (Cambridge Isotope Laboratories) were used as received. ${ }^{1} \mathrm{H}$ NMR spectra were recorded on Varian $300 \mathrm{MHz}$ and Varian $500 \mathrm{MHz}$ instruments, with shifts reported relative to the residual solvent peak. ${ }^{13} \mathrm{C}$ NMR spectra were recorded on a Varian $500 \mathrm{MHz}$ instrument, with shifts reported relative to the residual solvent peak. ${ }^{19} \mathrm{~F}$ NMR spectra were recorded on a Varian $300 \mathrm{MHz}$ instrument, with shifts reported relative to external $\mathrm{CFCl}_{3}$. Gas chromatographic analyses were performed on an Agilent Technologies Model 5973N Gas chromatograph/Mass spectrometer equipped with a Rtx-1 column. Melting points, uncorrected, were measured using Mel-Temp II instruments. Elemental analyses were performed by Atlantic Microlabs Inc., Norcross, GA. 
Reported yields in the text represent an average of two independent runs in which product was isolated in $\geq 95 \%$ purity as judged by a combination of ${ }^{1} \mathrm{H}$ NMR and GC-MS. Characterization data for each compound are reported for a single run; the isolated yield for each run may differ slightly from the average yields reported in the text (Tables 1-3).

General procedure for the deprotonation of pyrroles: In a nitrogen glovebox, $\mathrm{NaH}$ ( 2 equiv) was placed in a pear-shaped Schlenk flask, which was capped with a septum and removed from the glovebox. The flask was then connected to a Schlenk line, placed under vacuum for 30 min, and backfilled with argon. THF (anhydrous, 10-20 mL) was added via syringe. With a mercury bubbler open to relieve excess $\mathrm{H}_{2}$ pressure, the pyrrole was added dropwise via syringe. (In the case of 2-(2'-methoxyphenylpyrrole, a solution in anhydrous THF was prepared under argon and transferred via cannula to the $\mathrm{NaH}$ suspension.) After several hours, excess $\mathrm{NaH}$ was removed by Schlenk-filtration under an argon atmosphere. The filtrate was concentrated and dried in vacuo for roughly 12 hours, affording the pyrrolylsodium as a light-colored solid (yield typically $>90 \%$ ), which was stored in the glovebox.

\section{Procedures for palladium-catalyzed cross-coupling:}

General Procedure A: In a nitrogen glovebox, the pyrrolylsodium and $\mathrm{ZnCl}_{2}$ were weighed and transferred to a resealable 15-mL Schlenk tube containing a magnetic stirbar. The tube was then capped with a Teflon screwcap, removed from the glovebox, and connected to a Schlenk line. The atmosphere of the tube was evacuated, and replaced with argon. The Teflon screwcap was replaced under a flow of argon with a rubber septum. THF was added, via syringe; the septum was replaced with the Teflon screwcap, and the reaction mixture was stirred magnetically for about 10 minutes. The screwcap was removed under a flow of argon, which was then halted while the solid palladium precatalyst and phosphine supporting ligand, weighed out under air, were added as quickly as possible. The tube was then fitted with a septum and a needle extending below the tube neck, and purged under a 
flow of argon for about $5 \mathrm{~min}$. The aryl halide, if liquid, was then added via syringe, and the septum and needle were replaced with the Teflon screwcap. Solid aryl halides were added in the same manner as the precatalyst and ligand, and the tube was purged as before for 5 min prior to capping. The reaction vessel was then heated in an oil bath as described in the individual entries.

General procedure B: $\mathrm{NaH}$ dispersion was weighed out in air and placed in a $15-\mathrm{mL}$ resealable Schlenk tube, equipped with a Teflon screwcap and magnetic stirbar and connected to a Schlenk line. The atmosphere in the tube was evacuated, and replaced with argon; the screwcap was removed under a flow of argon and replaced with a rubber septum. THF $(\sim 0.5 \mathrm{~mL})$ was added via syringe, followed by pyrrole. The septum was replaced with the Teflon screwcap, left slightly open to allow relief of $\mathrm{H}_{2}$ pressure through the Schlenk line and a mercury bubbler. After the effervescence had ceased (several hours), the screwcap was replaced under a flow of argon with a rubber septum, and a solution of $\mathrm{ZnCl}_{2}$ (anhydrous, $0.50 \mathrm{M}$ in THF) ${ }^{1}$ was added via syringe. Roughly 10 minutes later, the palladium precatalyst and phosphine ligand were added, and the tube was purged, as described in Procedure A. The aryl halide was then added as described in procedure A. The reaction vessel was then capped with a Teflon screwcap and heated in an oil bath as described in the individual entries.

General procedure for workup: After the reaction was complete, the reaction mixture was cooled to room temperature. The tube was opened, and $\mathrm{Et}_{2} \mathrm{O}(\sim 10 \mathrm{~mL})$ and water $(\sim 10 \mathrm{~mL})$ were added. The resulting mixture was transferred to a separatory funnel, and the phases were separated. The aqueous layer was extracted with $\mathrm{Et}_{2} \mathrm{O}(2 \times 5 \mathrm{~mL})$; the combined organic layers were dried over $\mathrm{MgSO}_{4}$, filtered, and concentrated in vacuo. The crude product was purified by column chromatography on silica gel.

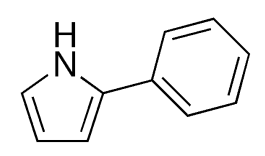

2-Phenyl-1H-pyrrole. General procedure A was followed using iodobenzene $(0.112 \mathrm{~mL}, 1.00 \mathrm{mmol})$, pyrrolylsodium (0.267 g, $3.00 \mathrm{mmol}), \mathrm{ZnCl}_{2}(0.409 \mathrm{~g}, 3.00 \mathrm{mmol}), \mathrm{Pd}(\mathrm{OAc})_{2}(1.1 \mathrm{mg}, 0.005 \mathrm{mmol}, 0.5$ 
mol \%), 2-(di-tert-butylphosphino)biphenyl (1.5 mg, $0.005 \mathrm{mmol}, 0.50 \mathrm{~mol} \%)$, and THF (1 mL). The reaction was run for 21 hours at $100{ }^{\circ} \mathrm{C}$. Standard workup and isolation, using 95:5 hexanes:ethyl acetate as eluant, afforded the title compound as a light red solid, $0.129 \mathrm{~g}(91 \%)$ : $\mathrm{mp} 128-130{ }^{\circ} \mathrm{C}$ (lit. ${ }^{2}$ 129-130 $\left.{ }^{\circ} \mathrm{C}\right) ;{ }^{1} \mathrm{H}$ NMR $\left(500 \mathrm{MHz}\right.$, acetone- $\left.d_{6}\right) \delta 10.67($ br s, $1 \mathrm{H}), 7.62(\mathrm{~d}, J=7.6 \mathrm{~Hz}, 2 \mathrm{H}), 7.32(\mathrm{t}, J=$ $7.6 \mathrm{~Hz}, 2 \mathrm{H}), 7.13(\mathrm{t}, J=7.3 \mathrm{~Hz}, 1 \mathrm{H}), 6.84(\mathrm{~m}, 1 \mathrm{H}), 6.51(\mathrm{~m}, 1 \mathrm{H}), 6.15(\mathrm{~m}, 1 \mathrm{H}) ;{ }^{13} \mathrm{C} \mathrm{NMR}(500 \mathrm{MHz}$ acetone- $\left.d_{6}\right) \delta 134.4,132.6,129.6,126.4,124.4,120.0,110.0,106.5 ; \mathrm{IR}\left(\mathrm{KBr}, \mathrm{cm}^{-1}\right) 3432,3393,3038$, 1604, 1496, 1466, 757, 718, 691. Anal. Calcd for $\mathrm{C}_{10} \mathrm{H}_{9} \mathrm{~N}$ : C, 83.88; H, 6.34. Found: C, 84.07; H, 6.34.

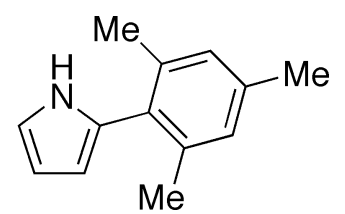

2-(2',4',6'-Trimethylphenyl)-1H-pyrrole. General procedure A was followed, except for the use of a 50-mL resealable Schlenk tube as the reaction vessel, using 2-bromomesitylene (1.25 $\mathrm{mL}, 8.17 \mathrm{mmol})$, pyrrolylsodium $(2.320 \mathrm{~g}, 26.0 \mathrm{mmol}), \mathrm{ZnCl}_{2}(3.560 \mathrm{~g}, 26.1 \mathrm{mmol}), \mathrm{Pd}_{2} \mathrm{dba}_{3}(18.7 \mathrm{mg}, 0.020 \mathrm{mmol}, 0.25$ mol \%), 2-(di-tert-butylphosphino)biphenyl (12.2 mg, $0.041 \mathrm{mmol}, 0.50 \mathrm{~mol} \%)$, and THF (20 mL). The reaction was run for 44 hours at $100{ }^{\circ} \mathrm{C}$. Standard workup and isolation, using 90:10 hexanes:ethyl acetate as eluant, afforded the title compound as a pale pink solid, $1.360 \mathrm{~g}(90 \%): \mathrm{mp} 99-100{ }^{\circ} \mathrm{C} ;{ }^{1} \mathrm{H}$ NMR (300 MHz, $\left.\mathrm{CDCl}_{3}\right) \delta 7.90$ (br s, $\left.1 \mathrm{H}\right), 6.95$ (s, $\left.2 \mathrm{H}\right), 6.86(\mathrm{~m}, 1 \mathrm{H}), 6.32(\mathrm{~m}, 1 \mathrm{H}), 6.07$ (m, $\left.1 \mathrm{H}\right)$, $2.33(\mathrm{~s}, 3 \mathrm{H}), 2.14(\mathrm{~m}, 6 \mathrm{H}) ;{ }^{13} \mathrm{C} \mathrm{NMR}\left(500 \mathrm{MHz}, \mathrm{CDCl}_{3}\right) \delta 138.8,137.8,130.9,129.7,128.3,117.0$ 108.6, 108.4, 21.3, 20.7; IR (KBr, cm $\left.{ }^{-1}\right) 3375,2968,2916,1455,1099,1025,852,794,576$. Anal. Calcd for $\mathrm{C}_{13} \mathrm{H}_{15} \mathrm{~N}$ : C, 84.28; H, 8.16. Found: C, 84.31; H, 8.26.

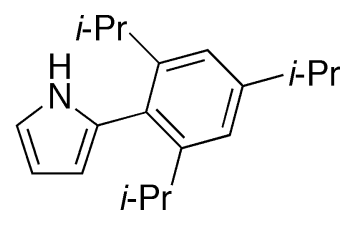

2-(2',4',6'-Triisopropylphenyl)-1H-pyrrole. General procedure A was followed using 1-bromo-2,4,6triisopropylbenzene $(0.180 \mathrm{~mL}, 1.00 \mathrm{mmol})$, pyrrolylsodium $(0.356 \mathrm{~g}, 4.00 \mathrm{mmol}), \mathrm{ZnCl}_{2}(0.546 \mathrm{~g}, 4.00$ 
mmol), $\mathrm{Pd}_{2} \mathrm{dba}_{3}(18.0 \mathrm{mg}, 0.020 \mathrm{mmol}, 2.0 \mathrm{~mol} \%$ ), 2-(dicyclohexylphosphino)biphenyl (14.6 mg, $0.042 \mathrm{mmol}, 4.0 \mathrm{~mol} \%)$, and THF $(1 \mathrm{~mL})$. The reaction was run for 41 hours at $100{ }^{\circ} \mathrm{C}$. Standard workup and isolation, using 95:5 hexanes:ethyl acetate as eluant, afforded the title compound as a pale brown solid, $0.189 \mathrm{~g}(70 \%)$ : mp $143-144{ }^{\circ} \mathrm{C} ;{ }^{1} \mathrm{H}$ NMR $\left(500 \mathrm{MHz}, \mathrm{CDCl}_{3}\right) \delta 7.95$ (br s, $\left.1 \mathrm{H}\right), 7.08(\mathrm{~s}, 2$ H), $6.86(\mathrm{~m}, 1 \mathrm{H}), 6.33(\mathrm{~m}, 1 \mathrm{H}), 6.11(\mathrm{~m}, 1 \mathrm{H}), 2.97$ (sept, $J=6.8 \mathrm{~Hz}, 1 \mathrm{H}), 2.75$ (sept, $J=6.8 \mathrm{~Hz}, 2 \mathrm{H})$, $1.33(\mathrm{~d}, J=6.8 \mathrm{~Hz}, 6 \mathrm{H}), 1.16(\mathrm{~d}, J=6.8 \mathrm{~Hz}, 12 \mathrm{H}) ;{ }^{13} \mathrm{C} \mathrm{NMR}\left(500 \mathrm{MHz}, \mathrm{CDCl}_{3}\right) \delta 149.8,149.4,129.2$, $128.9,120.8,116.8,109.2,108.5,34.6,30.8,24.8,24.3 ; \mathrm{IR}\left(\mathrm{KBr}, \mathrm{cm}^{-1}\right) 3424,2960,2925,2866,1458$ 882, 721. Anal. Calcd for $\mathrm{C}_{19} \mathrm{H}_{27} \mathrm{~N}$ : C, 84.70; H, 10.10. Found: C, 84.38; H, 10.10.

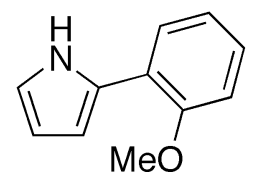

2-(2'-Methoxyphenyl)-1H-pyrrole. General procedure A was followed using 2-chloroanisole (0.127 $\mathrm{mL}, 1.000 \mathrm{mmol})$, pyrrolylsodium $(0.267 \mathrm{~g}, 3.00 \mathrm{mmol}), \mathrm{ZnCl}_{2}(0.409 \mathrm{~g}, 3.00 \mathrm{mmol}), \operatorname{Pd}(\mathrm{OAc})_{2}(1.1$ mg, $0.005 \mathrm{mmol}, 0.5 \mathrm{~mol} \%$ ), 2-(di-tert-butylphosphino)biphenyl (1.5 mg, $0.005 \mathrm{mmol}, 0.5 \mathrm{~mol} \%$ ), and THF $(1 \mathrm{~mL})$. The reaction was run for 25 hours at $60^{\circ} \mathrm{C}$. Standard workup and isolation, using 80:20 hexanes:ethyl acetate as eluant, afforded the title compound as a light yellow solid, $0.157 \mathrm{~g}(91 \%)$ : $\mathrm{mp}$ 65-66 ${ }^{\circ} \mathrm{C}\left(\right.$ lit. $^{3}$ 66-67 $\left.{ }^{\circ} \mathrm{C}\right) ;{ }^{1} \mathrm{H}$ NMR $\left(500 \mathrm{MHz}, \mathrm{CDCl}_{3}\right) \delta 9.90$ (br s, $\left.1 \mathrm{H}\right), 7.77(\mathrm{dd}, J=7.8 \mathrm{~Hz}$ and 1.7 $\mathrm{Hz}, 1 \mathrm{H}$ ), 7.24 (distorted td, $J=7.8 \mathrm{~Hz}$ and $1.7 \mathrm{~Hz}, 1 \mathrm{H}$ ), 7.08 (distorted td, $J=7.8 \mathrm{~Hz}$ and $0.6 \mathrm{~Hz}, 1 \mathrm{H}$ ), $7.03(\mathrm{~d}, J=8.4 \mathrm{~Hz}, 1 \mathrm{H}), 6.94-6.96(\mathrm{~m}, 1 \mathrm{H}), 6.73-6.75(\mathrm{~m}, 1 \mathrm{H}), 6.39-6.41(\mathrm{~m}, 1 \mathrm{H}), 4.00(\mathrm{~s}, 3 \mathrm{H}) ;{ }^{13} \mathrm{C}$ $\mathrm{NMR}\left(500 \mathrm{MHz}, \mathrm{CDCl}_{3}\right) \delta 154.8,129.9,126.8,126.7,121.5,121.2,117.9,111.7,108.9,106.2,55.7$; IR $\left(\mathrm{KBr}, \mathrm{cm}^{-1}\right) 3443,1493,1235,1112,1023,754,726$.

Note: We have been unable to obtain satisfactory elemental analyses for this compound. The ${ }^{1} \mathrm{H}$ and ${ }^{13} \mathrm{C}$ NMR spectra are reproduced below as a measure of purity. 

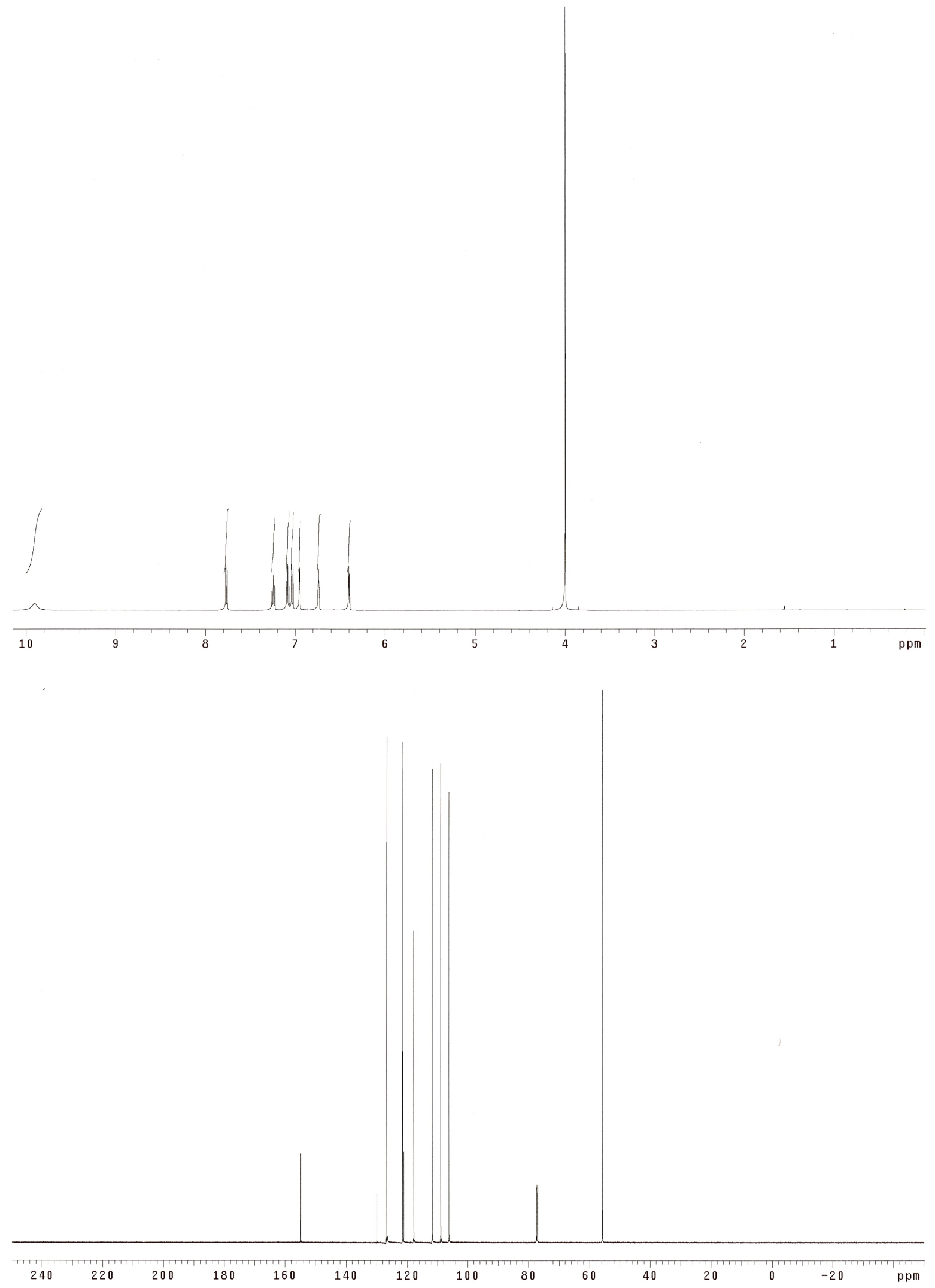

Figure S1. ${ }^{1} \mathrm{H}$ and ${ }^{13} \mathrm{C}$ NMR of 2-(2'-methoxyphenyl)-1H-pyrrole in $\mathrm{CDCl}_{3}$. 


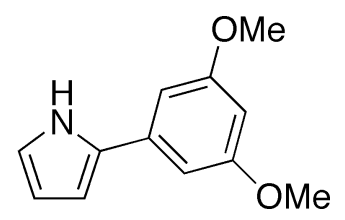

2-(3',5'-Dimethoxyphenyl)-1H-pyrrole. General procedure A was followed using 3,5dimethoxychlorobenzene $(0.173 \mathrm{~g}, 1.00 \mathrm{mmol})$, pyrrolylsodium $(0.142 \mathrm{~g}, 1.60 \mathrm{mmol}), \mathrm{ZnCl}_{2}(0.218 \mathrm{~g}$, $1.60 \mathrm{mmol}), \mathrm{Pd}(\mathrm{OAc})_{2}(1.1 \mathrm{mg}, 0.005 \mathrm{mmol}, 0.5 \mathrm{~mol} \%)$, 2-(di-tert-butylphosphino)biphenyl (1.5 mg, $0.005 \mathrm{mmol}, 0.5 \mathrm{~mol} \%)$, and THF $(1 \mathrm{~mL})$. The reaction was run for 23 hours at $60{ }^{\circ} \mathrm{C}$. Standard workup and isolation, using 80:20 hexanes:ethyl acetate as eluant, afforded the title compound as a pale orange solid, $0.159 \mathrm{~g} \mathrm{(78 \% ):} \mathrm{mp} 77-78{ }^{\circ} \mathrm{C} ;{ }^{1} \mathrm{H}$ NMR $\left(500 \mathrm{MHz}, \mathrm{CDCl}_{3}\right) \delta 8.46$ (br s, $\left.1 \mathrm{H}\right), 6.86(\mathrm{~s}, 1 \mathrm{H})$, $6.64(\mathrm{~s}, 2 \mathrm{H}), 6.53(\mathrm{~m}, 1 \mathrm{H}), 6.36(\mathrm{~m}, 1 \mathrm{H}), 6.31(\mathrm{~m}, 1 \mathrm{H}), 3.84(\mathrm{~s}, 6 \mathrm{H}) ;{ }^{13} \mathrm{C} \mathrm{NMR}\left(500 \mathrm{MHz}, \mathrm{CDCl}_{3}\right) \delta$ 161.4, 134.9, 132.2, 119.1, 110.2, 106.5, 102.5, 98.4, 55.6; IR $\left(\mathrm{KBr}, \mathrm{cm}^{-1}\right) 3398,1597,1475,1247$, 1195, 1156, 1063, 835, 718. Anal. Calcd for $\mathrm{C}_{12} \mathrm{H}_{13} \mathrm{NO}_{2}$ : C, 70.92; H, 6.45. Found: C, 70.54; H, 6.44 .

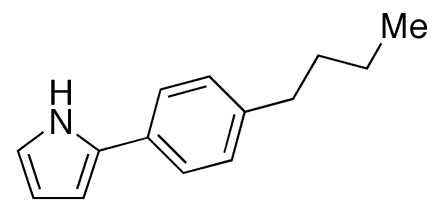

2-(4'-n-Butylphenyl)-1H-pyrrole. General procedure A was followed using 4-(n-butyl)-chlorobenzene (0.169 mL, $1.00 \mathrm{mmol})$, pyrrolylsodium (0.142 g, $1.60 \mathrm{mmol}), \mathrm{ZnCl}_{2}(0.218 \mathrm{~g}, 1.60 \mathrm{mmol}), \mathrm{Pd}(\mathrm{OAc})_{2}$ (1.1 mg, $0.005 \mathrm{mmol}, 0.5 \mathrm{~mol} \%$ ), 2-(di-tert-butylphosphino)biphenyl (1.5 mg, $0.005 \mathrm{mmol}, 0.5 \mathrm{~mol}$ $\%)$, and THF $(1 \mathrm{~mL})$. The reaction was run for 16 hours at $60{ }^{\circ} \mathrm{C}$. Standard workup and isolation, using 95:5 hexanes:ethyl acetate as eluant, afforded the title compound as an amorphous brown-orange solid, $0.136 \mathrm{~g}(68 \%): \mathrm{mp} 104-107{ }^{\circ} \mathrm{C}(\mathrm{dec}.) ;{ }^{1} \mathrm{H}$ NMR $\left(500 \mathrm{MHz}, \mathrm{CDCl}_{3}\right) \delta 8.39$ (br s, $\left.1 \mathrm{H}\right), 7.42$ (d, $J=8.1$ $\mathrm{Hz}, 2 \mathrm{H}), 7.22(\mathrm{~d}, J=8.1 \mathrm{~Hz}, 2 \mathrm{H}), 6.85(\mathrm{~d}, J=1.2 \mathrm{~Hz}, 1 \mathrm{H}), 6.54(\mathrm{~s}, 1 \mathrm{H}), 6.34(\mathrm{q}, J=2.8 \mathrm{~Hz}, 1 \mathrm{H})$, $2.66(\mathrm{t}, J=7.6 \mathrm{~Hz}, 2 \mathrm{H}), 1.67$ (quint, $J=7.6 \mathrm{~Hz}, 2 \mathrm{H}), 1.43$ (sext, $J=7.3 \mathrm{~Hz}, 2 \mathrm{H}$ ), 1.00 (t, $J=7.3 \mathrm{~Hz}, 3$ $\mathrm{H}) ;{ }^{13} \mathrm{C}$ NMR (500 MHz, $\left.\mathrm{CDCl}_{3}\right) \delta$ 141.1, 132.4, 130.4, 129.1, 124.0, 118.7, 110.1, 105.5, 35.5, 33.8, 
22.6, 14.2; IR (KBr, cm $\left.{ }^{-1}\right)$ 3432, 2958, 2928, 2857, 1509, 1458, 1034, 794, 713. Anal. Calcd for $\mathrm{C}_{14} \mathrm{H}_{17} \mathrm{~N}: \mathrm{C}, 84.37 ; \mathrm{H}, 8.60$. Found: C, 84.29; H, 8.69.

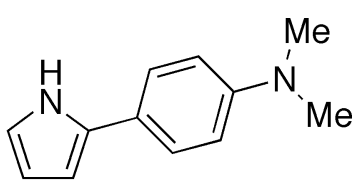

\section{2-(4'-Dimethylaminophenyl)-1H-pyrrole.}

General procedure A: followed using 4-bromo- $N, N$-dimethylaniline $(0.200 \mathrm{~g}, 1.00 \mathrm{mmol})$, pyrrolylsodium (0.142 g, $1.60 \mathrm{mmol}), \mathrm{ZnCl}_{2}(0.218 \mathrm{~g}, 1.60 \mathrm{mmol}), \mathrm{Pd}(\mathrm{OAc})_{2}(1.1 \mathrm{mg}, 0.005 \mathrm{mmol}, 0.5$ mol \%), 2-(di-tert-butylphosphino)biphenyl (1.5 mg, $0.005 \mathrm{mmol}, 0.5 \mathrm{~mol} \%)$, and THF (1 mL). The reaction was run for 19 hours at $60{ }^{\circ} \mathrm{C}$. Standard workup and isolation, using 50:50 hexane:diethyl ether as eluant, afforded the title compound as a light yellow solid (which after several hours' exposure to air forms a dark blue-violet material), $0.096 \mathrm{~g}(52 \%)$.

General procedure B: followed using pyrrole $(0.111 \mathrm{~mL}, 1.6 \mathrm{mmol}), \mathrm{NaH}(60 \%$ dispersion in mineral oil, $0.060 \mathrm{~g}, 1.5 \mathrm{mmol}), \mathrm{ZnCl}_{2}$ solution $(0.50 \mathrm{M}$ in $\mathrm{THF}, 3.2 \mathrm{~mL}, 1.6 \mathrm{mmol})$, and the same quantities of aryl bromide, precatalyst and ligand as in Procedure A. The reaction was allowed to run for 17 hours at $60{ }^{\circ} \mathrm{C}$; isolation and purification as described above afforded $0.110 \mathrm{~g}(60 \%)$ of the title compound: $\mathrm{mp} 167-169{ }^{\circ} \mathrm{C}$ (dec.) (lit. ${ }^{4} 160{ }^{\circ} \mathrm{C}$, dec.); ${ }^{1} \mathrm{H} \mathrm{NMR}\left(500 \mathrm{MHz}, \mathrm{CDCl}_{3}\right) \delta 8.30$ (br s, $\left.1 \mathrm{H}\right)$, $7.38(\mathrm{~d}, J=8.8 \mathrm{~Hz}, 2 \mathrm{H}), 6.76(\mathrm{~d}, J=8.8 \mathrm{~Hz}, 2 \mathrm{H}), 6.81(\mathrm{~m}, 1 \mathrm{H}), 6.37(\mathrm{~m}, 1 \mathrm{H}), 6.28(\mathrm{~m}, 1 \mathrm{H}), 2.98(\mathrm{~s}$, $6 \mathrm{H}) ;{ }^{13} \mathrm{C} \mathrm{NMR}\left(500 \mathrm{MHz}, \mathrm{CDCl}_{3}\right) \delta 149.4,133.1,125.3,122.0,117.7,113.1,109.9,104.1,40.8 ;$ IR $\left(\mathrm{KBr}, \mathrm{cm}^{-1}\right) 3421,2879,2798,1615,1517,818$. Anal. Calcd for $\mathrm{C}_{12} \mathrm{H}_{14} \mathrm{~N}_{2}$ : C, 77.38; H, 7.58. Found: C, 77.13; H, 7.36. 


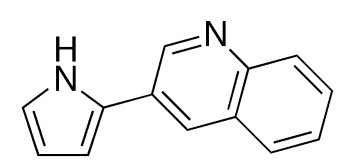

2-(3'-Quinolyl)-1H-pyrrole. General procedure A was followed using 3-bromoquinoline $(0.136 \mathrm{~mL}$, $1.00 \mathrm{mmol})$, pyrrolylsodium $(0.142 \mathrm{~g}, 1.60 \mathrm{mmol}), \mathrm{ZnCl}_{2}(0.218 \mathrm{~g}, 1.60 \mathrm{mmol}), \mathrm{Pd}(\mathrm{OAc})_{2}(1.1 \mathrm{mg}$, $0.005 \mathrm{mmol}, 0.5 \mathrm{~mol} \%$ ), 2-(Di-tert-butylphosphino)biphenyl (1.5 mg, $0.005 \mathrm{mmol}, 0.5 \mathrm{~mol} \%$ ), and THF $(1 \mathrm{~mL})$. The reaction was run for 18 hours at $100{ }^{\circ} \mathrm{C}$. Standard workup and isolation, using 50:50 hexanes:ethyl acetate as eluant, afforded the title compound as a yellow solid, $0.142 \mathrm{~g} \mathrm{(73 \% ):} \mathrm{mp}$ 172-173 ${ }^{\circ} \mathrm{C}\left(\right.$ lit. $\left.^{5} 174-175{ }^{\circ} \mathrm{C}\right) ;{ }^{1} \mathrm{H}$ NMR $\left(500 \mathrm{MHz}\right.$, acetone- $\left.d_{6}\right) \delta 10.90$ (br s, $\left.1 \mathrm{H}\right), 9.27(\mathrm{~d}, J=1.8 \mathrm{~Hz}$, $1 \mathrm{H}), 8.39(\mathrm{~d}, J=1.8 \mathrm{~Hz}, 1 \mathrm{H}), 8.00(\mathrm{~d}, J=8.2 \mathrm{~Hz}, 1 \mathrm{H}), 7.86(\mathrm{~d}, J=8.2 \mathrm{~Hz}, 1 \mathrm{H}), 7.64(\mathrm{td}, J=7.7 \mathrm{~Hz}$ and $1.5 \mathrm{~Hz}, 1 \mathrm{H}), 7.54(\mathrm{td}, \mathrm{J}=7.6 \mathrm{~Hz}$ and $1.2 \mathrm{~Hz}, 1 \mathrm{H}), 7.02(\mathrm{~m}, 1 \mathrm{H}), 6.83(\mathrm{~m}, 1 \mathrm{H}), 6.28(\mathrm{~m}, 1 \mathrm{H}) ;{ }^{13} \mathrm{C}$ NMR $\left(500 \mathrm{MHz}\right.$, acetone- $\left.d_{6}\right) \delta 148.9,147.5,130.1,129.7,129.3,129.1,128.6,128.0,127.9,127.5$, 121.5, 110.8, 108.3; IR (KBr, cm $\left.{ }^{-1}\right) 3092$ (broad), 1617, 1561, 1497, 1477, 1425, 1125, 1114, 780, 711. Anal. Calcd for $\mathrm{C}_{13} \mathrm{H}_{10} \mathrm{~N}_{2}$ : C, 80.39; H, 5.19. Found: C, 80.27; H, 5.35.

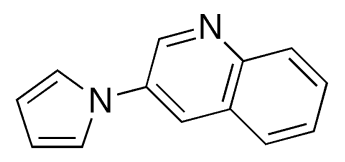

$N$-(3'-Quinolyl)pyrrole. General procedure A was followed using 3-bromoquinoline (0.136 mL, 1.00 mmol), pyrrolylsodium (0.142 g, $1.60 \mathrm{mmol}), \mathrm{ZnCl}_{2}(0.218 \mathrm{~g}, 1.60 \mathrm{mmol}), \mathrm{Pd}(\mathrm{OAc})_{2}(1.1 \mathrm{mg}, 0.005$ mmol, $0.5 \mathrm{~mol} \%$ ), 2-(dicyclohexylphosphino)biphenyl (1.8 $\mathrm{mg}, 0.005 \mathrm{mmol}, 0.5 \mathrm{~mol} \%$ ), and THF (1 $\mathrm{mL}$ ). The reaction was run for 16 hours at $60{ }^{\circ} \mathrm{C}$. Standard workup and isolation, using 80:20 hexanes:ethyl acetate as eluant, afforded the title compound as a pale yellow solid, $0.133 \mathrm{~g} \mathrm{(69 \% ):} \mathrm{mp}$ 86-87 ${ }^{\circ} \mathrm{C} ;{ }^{1} \mathrm{H}$ NMR $\left(500 \mathrm{MHz}, \mathrm{CDCl}_{3}\right) \delta 9.09(\mathrm{~d}, J=2.6 \mathrm{~Hz}, 1 \mathrm{H}), 8.14(\mathrm{dd}, J=8.5 \mathrm{~Hz}$ and $0.4 \mathrm{~Hz}, 1$ H), $8.02(\mathrm{~d}, J=2.6 \mathrm{~Hz}, 1 \mathrm{H}), 7.82(\mathrm{dd}, J=8.1 \mathrm{~Hz}$ and $0.5 \mathrm{~Hz}, 1 \mathrm{H}), 7.69(\mathrm{dtd}, J=7.7 \mathrm{~Hz}$ and $1.5 \mathrm{~Hz}$ and $1.5 \mathrm{~Hz}, 1 \mathrm{H}), 7.58(\mathrm{dtd}, J=7.5 \mathrm{~Hz}$ and $1.3 \mathrm{~Hz}$ and $1.2 \mathrm{~Hz}, 1 \mathrm{H}), 7.22(\mathrm{t}, J=2.1 \mathrm{~Hz}, 2 \mathrm{H}), 6.46$ (t, $J=2.1$ $\mathrm{Hz}, 2 \mathrm{H}) ;{ }^{13} \mathrm{C} \mathrm{NMR}\left(500 \mathrm{MHz}, \mathrm{CDCl}_{3}\right) \delta$ 146.4, 144.7, 134.1, 129.5, 129.1, 128.1, 127.8, 127.6, 124.7, 
119.6, 111.7; IR (KBr, $\left.\mathrm{cm}^{-1}\right)$ 3128, 3103, 1494, 1356, 1300, 734. Anal. Calcd for $\mathrm{C}_{13} \mathrm{H}_{10} \mathrm{~N}_{2}$ : C, 80.39; H, 5.19. Found: C, 80.16; H, 5.09.

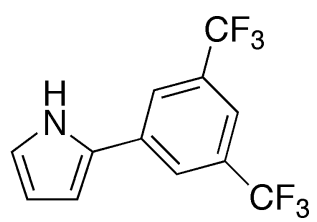

\section{2-[3',5'-Bis(trifluoromethyl)phenyl]-1H-pyrrole.}

General procedure A: followed using 3,5-bis(trifluoromethyl)bromobenzene $(0.172 \mathrm{~mL}, 1.00$ mmol), pyrrolylsodium (0.267 g, $3.00 \mathrm{mmol}, 3.0$ equivalents), $\mathrm{ZnCl}_{2}$ (0.409 g, $\left.3.00 \mathrm{mmol}\right), \mathrm{Pd}(\mathrm{OAc})_{2}$ (1.1 mg, $0.005 \mathrm{mmol}, 0.5 \mathrm{~mol} \%$ ), 2-(dicyclohexylphosphino)biphenyl (1.8 mg, $0.005 \mathrm{mmol}, 0.5 \mathrm{~mol}$ $\%$ ), and THF $(1 \mathrm{~mL})$. The reaction was run for 18 hours at $80^{\circ} \mathrm{C}$. Standard workup and isolation, using 90:10 hexanes:ethyl acetate as eluant, afforded the title compound as a pale yellow solid, $0.263 \mathrm{~g}(94 \%)$.

General procedure B: followed using pyrrole $(0.139 \mathrm{~mL}, 2.0 \mathrm{mmol}), \mathrm{NaH}(60 \%$ dispersion in mineral oil, $0.080 \mathrm{~g}, 2.0 \mathrm{mmol}), \mathrm{ZnCl}_{2}$ solution $(0.50 \mathrm{M}$ in $\mathrm{THF}, 4.0 \mathrm{~mL}, 2.0 \mathrm{mmol})$, and the same quantities of aryl bromide, precatalyst and ligand as in Procedure A. The reaction was allowed to run for 25 hours at $80^{\circ} \mathrm{C}$. Isolation and purification as described above afforded $0.202 \mathrm{~g}(72 \%)$ of the title compound: $\mathrm{mp} 64-65{ }^{\circ} \mathrm{C} ;{ }^{1} \mathrm{H}$ NMR $\left(500 \mathrm{MHz}, \mathrm{CDCl}_{3}\right) \delta 8.58$ (br s, $\left.1 \mathrm{H}\right), 7.85(\mathrm{~s}, 2 \mathrm{H}), 7.68(\mathrm{~s}, 1 \mathrm{H})$, $6.97(\mathrm{~m}, 1 \mathrm{H}), 6.69(\mathrm{~m}, 1 \mathrm{H}), 6.37(\mathrm{~m}, 1 \mathrm{H}) ;{ }^{13} \mathrm{C} \mathrm{NMR}\left(500 \mathrm{MHz}, \mathrm{CDCl}_{3}\right) \delta 134.8,132.4(\mathrm{q}, J=33.2$ $\mathrm{Hz}), 129.4,123.6(\mathrm{q}, J=272.8 \mathrm{~Hz}), 123.5,121.1,119.4(\mathrm{~m}, J=3.8 \mathrm{~Hz}), 111.2,108.8 ;{ }^{19} \mathrm{~F}$ NMR $(300$ $\left.\mathrm{MHz}, \mathrm{CDCl}_{3}\right) \delta-63.4$; IR (KBr, cm $\left.{ }^{-1}\right)$ 3489, 3395, 1474, 1375, 1284, 1186, 1123, 1068, 895, 884, 744, 734, 705, 684. Anal. Calcd for $\mathrm{C}_{12} \mathrm{H}_{7} \mathrm{~F}_{6} \mathrm{~N}: \mathrm{C}, 51.63 ; \mathrm{H}, 2.53$. Found: C, 51.74; H, 2.42 . 


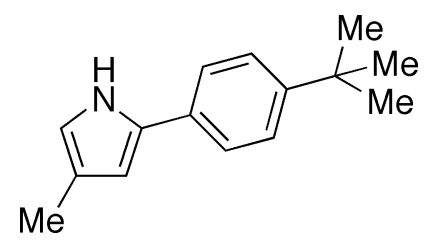

2-(4'-tert-Butylphenyl)-4-methyl-1H-pyrrole. General procedure A was followed using 4-tert-butylbromobenzene $(0.087 \mathrm{~mL}, 0.50 \mathrm{mmol}), 3$-methylpyrrolylsodium $(0.083 \mathrm{~g}, 0.80 \mathrm{mmol}), \mathrm{ZnCl}_{2}(0.109 \mathrm{~g}$, $0.800 \mathrm{mmol}), \mathrm{Pd}_{2} \mathrm{dba}_{3}(11.5 \mathrm{mg}, 0.013 \mathrm{mmol}, 2.5 \mathrm{~mol} \%$ ), 2-(di-tert-butylphosphino)biphenyl (7.5 mg, $0.025 \mathrm{mmol}, 5.0 \mathrm{~mol} \%)$, and THF $(1 \mathrm{~mL})$. The reaction was run for 43 hours at $100^{\circ} \mathrm{C}$. Standard workup and isolation, using 90:10 hexanes:ethyl acetate as eluant, afforded the title compound as a green solid, $0.071 \mathrm{~g}(67 \%)$ : $\mathrm{mp} 93-100{ }^{\circ} \mathrm{C}$ (dec.); ${ }^{1} \mathrm{H}$ NMR (300 MHz, $\left.\mathrm{CDCl}_{3}\right) \delta 8.04$ (br s, $\left.1 \mathrm{H}\right), 7.40$ $(\mathrm{s}, 4 \mathrm{H}), 6.62(\mathrm{~m}, 1 \mathrm{H}), 6.36(\mathrm{~m}, 1 \mathrm{H}), 2.19(\mathrm{~s}, 3 \mathrm{H}), 1.37(\mathrm{~s}, 9 \mathrm{H}) ;{ }^{13} \mathrm{C} \mathrm{NMR}\left(500 \mathrm{MHz}, \mathrm{CDCl}_{3}\right) \delta 149.2$ $132.3,130.4,125.9,123.7,120.7,116.6,107.2,34.7,31.5,12.2 ; \mathrm{IR}\left(\mathrm{KBr}, \mathrm{cm}^{-1}\right) 3457,2962,1523$, 1435, 1113, 835, 799, 559. Anal. Calcd for $\mathrm{C}_{15} \mathrm{H}_{19} \mathrm{~N}$ : C, 84.46; H, 8.98. Found: C, 84.43; H, 8.60.

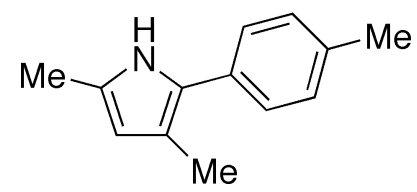

2-(p-Tolyl)-3,5-dimethyl-1H-pyrrole. General procedure A was followed using 4-bromotoluene $(0.222$ g, $1.30 \mathrm{mmol}), 2$,4-dimethylpyrrolylsodium (0.300 g, $2.56 \mathrm{mmol}), \mathrm{ZnCl}_{2}(0.348 \mathrm{~g}, 2.55 \mathrm{mmol})$, $\mathrm{Pd}(\mathrm{OAc})_{2}(14.4 \mathrm{mg}, 0.064 \mathrm{mmol}, 5.0 \mathrm{~mol} \%$ ), 2-(Dicyclohexylphosphino)biphenyl (22.2 mg, 0.063 mmol, $5.0 \mathrm{~mol} \%)$, and 1,4-dioxane $(1 \mathrm{~mL})$. The reaction was run for 37 hours at $100^{\circ} \mathrm{C}$. Standard workup and isolation, using 75:25 hexanes:ethyl acetate as eluant, afforded the title compound as a dark red solid, $0.151 \mathrm{~g}(63 \%)$ : $\mathrm{mp} 34-35{ }^{\circ} \mathrm{C} ;{ }^{1} \mathrm{H}$ NMR (500 MHz, $\left.\mathrm{CDCl}_{3}\right) \delta 7.77$ (br s, $\left.1 \mathrm{H}\right), 7.30(\mathrm{~d}, J=8.2$ $\mathrm{Hz}, 2 \mathrm{H}), 7.21(\mathrm{~d}, J=8.6 \mathrm{~Hz}, 2 \mathrm{H}), 5.84(\mathrm{~m}, 1 \mathrm{H}), 2.38(\mathrm{~s}, 3 \mathrm{H}), 2.30(\mathrm{~s}, 3 \mathrm{H}), 2.24(\mathrm{~s}, 3 \mathrm{H}) ;{ }^{13} \mathrm{C} \mathrm{NMR}$ $\left(500 \mathrm{MHz}, \mathrm{CDCl}_{3}\right) \delta 135.4,131.2,129.5,127.3,127.0,126.1,116.1,110.3,21.3,13.2,12.6 ; \mathrm{IR}(\mathrm{KBr}$ $\mathrm{cm}^{-1}$ ) 3416, 2920, 1528, 1259, 821, 789, 551. Anal. Calcd for $\mathrm{C}_{13} \mathrm{H}_{15} \mathrm{~N}: \mathrm{C}, 84.28 ; \mathrm{H}, 8.16$. Found: C, 84.28; H, 8.12. 


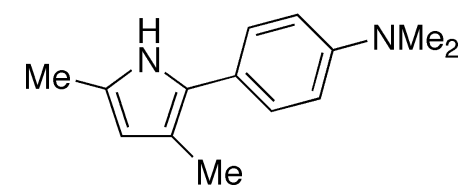

2-(4'-Dimethylaminophenyl)-3,5-dimethylpyrrole. General procedure A was followed using 4bromo- $N, N$-dimethylaniline (0.086 g, $0.43 \mathrm{mmol}), 2,4$-dimethylpyrrolylsodium $(0.100 \mathrm{~g}, 0.850 \mathrm{mmol})$, $\mathrm{ZnCl}_{2}(0.116 \mathrm{~g}, 0.850 \mathrm{mmol}), \mathrm{Pd}(\mathrm{OAc})_{2}(4.8 \mathrm{mg}, 0.021 \mathrm{mmol}, 5.0 \mathrm{~mol} \%)$, 2-(di-tert-butylphosphino)biphenyl $(6.4 \mathrm{mg}, 0.021 \mathrm{mmol}, 5.0 \mathrm{~mol} \%)$, and THF $(1 \mathrm{~mL})$. The reaction was run for 17 hours at 100 ${ }^{\circ} \mathrm{C}$. Standard workup and isolation, using 80:20 hexanes:ethyl acetate as eluant, afforded the title compound as a dark red solid, $0.070 \mathrm{~g}(76 \%)$ : $\mathrm{mp} 104-105{ }^{\circ} \mathrm{C} ;{ }^{1} \mathrm{H} \mathrm{NMR}\left(500 \mathrm{MHz}, \mathrm{CDCl}_{3}\right) \delta 7.69$ (br s, $1 \mathrm{H}), 7.21(\mathrm{~d}, J=8.9 \mathrm{~Hz}, 2 \mathrm{H}), 6.81(\mathrm{~d}, J=8.9 \mathrm{~Hz}, 2 \mathrm{H}), 6.51(\mathrm{~s}, 1 \mathrm{H}), 2.98(\mathrm{~s}, 6 \mathrm{H}), 2.26(\mathrm{~s}, 3 \mathrm{H}), 2.09$ (s, $3 \mathrm{H}) ;{ }^{13} \mathrm{C}$ NMR $\left(500 \mathrm{MHz}, \mathrm{CDCl}_{3}\right) \delta$ 148.7, 130.6, 124.9, 124.4, 121.4, 118.1, 113.6, 112.7, 41.0, 12.4, 11.4; IR $\left(\mathrm{KBr}, \mathrm{cm}^{-1}\right) 3341,1616,1535,1512,1351,819$.

Note: We have been unable to obtain satisfactory elemental analyses for this compound. The ${ }^{1} \mathrm{H}$ and ${ }^{13} \mathrm{C}$ NMR spectra are reproduced below as a measure of purity. 

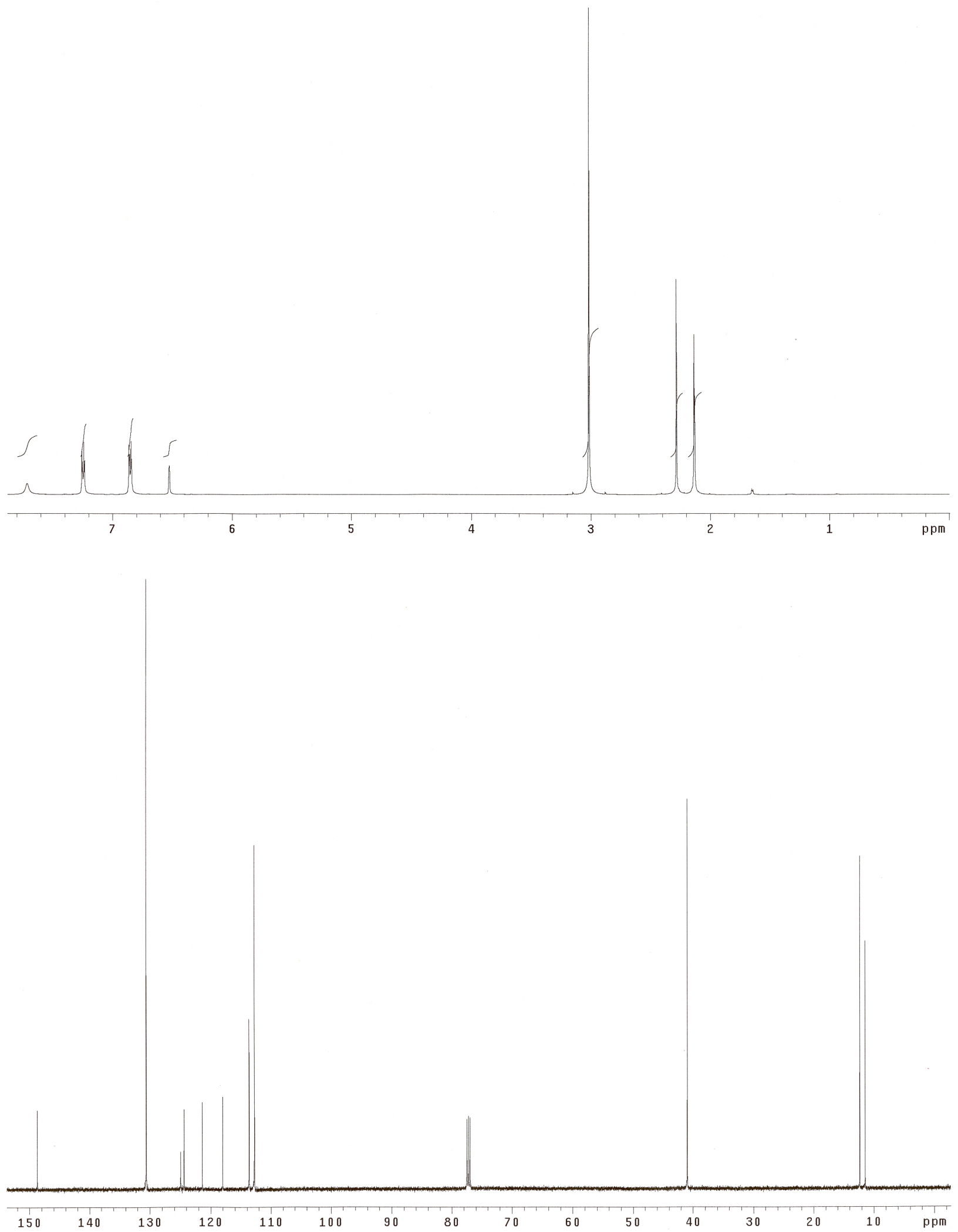

Figure S2. ${ }^{1} \mathrm{H}$ and ${ }^{13} \mathrm{C}$ NMR of 2-(4'-Dimethylaminophenyl)-3,5-dimethylpyrrole in $\mathrm{CDCl}_{3}$. 


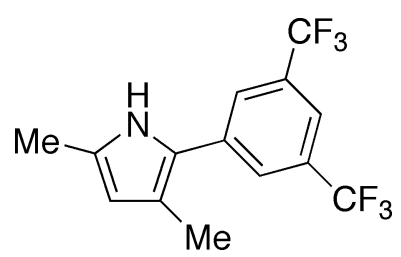

2-[3,5-Bis(trifluoromethyl)phenyl]-3,5-dimethyl-1H-pyrrole. General procedure A was followed using 3,5-bis(trifluoromethyl)bromobenzene $(0.296 \mathrm{~mL}, 1.72 \mathrm{mmol})$, 2,4-dimethylpyrrolylsodium (0.400 g, $3.410 \mathrm{mmol}), \mathrm{ZnCl}_{2}$ (0.464 g, $\left.3.4000 \mathrm{mmol}\right), \mathrm{Pd}(\mathrm{OAc})_{2}$ (19.2 mg, $0.085 \mathrm{mmol}, 5.0 \mathrm{~mol} \%$ ), $2-$ (dicyclohexylphosphino)-2',6'-dimethoxy-1,1'-biphenyl (35.2 mg, $0.085 \mathrm{mmol}, 5.0 \mathrm{~mol} \%$ ), and THF (4 $\mathrm{mL}$ ). The reaction was run for 54 hours at $100{ }^{\circ} \mathrm{C}$. Standard workup and isolation, using 90:10 hexanes:ethyl acetate as eluant, afforded the title compound as a red solid, $0.370 \mathrm{~g}(70 \%)$ : $\mathrm{mp} 45-46{ }^{\circ} \mathrm{C}$; ${ }^{1} \mathrm{H}$ NMR (500 MHz, $\left.\mathrm{CDCl}_{3}\right) \delta 7.93($ br s, $1 \mathrm{H}), 7.78$ (s, $\left.2 \mathrm{H}\right), 7.66(\mathrm{~s}, 1 \mathrm{H}), 5.89(\mathrm{~s}, 1 \mathrm{H}), 2.33(\mathrm{~s}, 3 \mathrm{H})$, 2.27 (s, $3 \mathrm{H}) ;{ }^{13} \mathrm{C}$ NMR $\left(500 \mathrm{MHz}, \mathrm{CDCl}_{3}\right) \delta$ 135.9, 132.3 (q, J = 33.0 Hz), 130.0, 125.2, 124.2, 123.7 $(\mathrm{q}, \mathrm{J}=272.9 \mathrm{~Hz}), 119.8,118.5(\mathrm{~m}, \mathrm{~J}=3.9 \mathrm{~Hz}), 111.7,13.2,12.8 ;{ }^{19} \mathrm{~F} \mathrm{NMR}\left(300 \mathrm{MHz}, \mathrm{CDCl}_{3}\right) \delta-63.4$; IR $\left(\mathrm{KBr}, \mathrm{cm}^{-1}\right) 3490,1367,1279,1171,1130,1089$. Anal. Calcd for $\mathrm{C}_{14} \mathrm{H}_{11} \mathrm{~F}_{6} \mathrm{~N}: \mathrm{C}, 54.73 ; \mathrm{H}, 3.61$. Found: C, 54.82; H, 3.63.

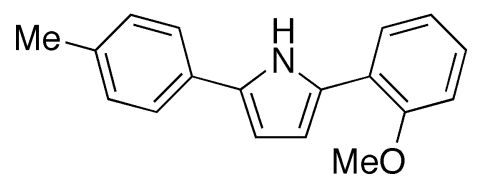

2-(2'-Methoxyphenyl)-5-(p-tolyl)-1H-pyrrole. General procedure A was followed using 4bromotoluene (0.058 g, $0.34 \mathrm{mmol}), 2$-(2'-methoxyphenyl)pyrrolylsodium (0.100 g, $0.513 \mathrm{mmol}), \mathrm{ZnCl}_{2}$ (0.070 g, $0.51 \mathrm{mmol}), \mathrm{Pd}(\mathrm{OAc})_{2}(3.8 \mathrm{mg}, 0.017 \mathrm{mmol}, 5.0 \mathrm{~mol} \%)$, 2-(di-tert-butylphosphino)-2'-(N,Ndimethylamino)biphenyl (5.8 $\mathrm{mg}, 0.017 \mathrm{mmol}, 5.0 \mathrm{~mol} \%)$, and THF (1 mL). The reaction was run for 24 hours at $100{ }^{\circ} \mathrm{C}$. Standard workup and isolation, using 75:25 hexanes:ethyl acetate as eluant, afforded the title compound as a pale orange solid, $0.070 \mathrm{~g}(78 \%)$ : $\mathrm{mp} 101-103{ }^{\circ} \mathrm{C} ;{ }^{1} \mathrm{H}$ NMR $(500 \mathrm{MHz}$, acetone- $\left.d_{6}\right) \delta 10.38(\mathrm{br} \mathrm{s}, 1 \mathrm{H}), 7.70(\mathrm{dd}, J=7.8 \mathrm{~Hz}$ and $1.7 \mathrm{~Hz}, 1 \mathrm{H}), 7.55(\mathrm{~d}, J=8.1 \mathrm{~Hz}, 2 \mathrm{H}), 7.18(\mathrm{~m}$, 
$2 \mathrm{H}), 7.10(\mathrm{~d}, J=8.2 \mathrm{~Hz}, 1 \mathrm{H}), 6.98(\mathrm{td}, J=7.5 \mathrm{~Hz}$ and $1.1 \mathrm{~Hz}, 2 \mathrm{H}), 6.68(\mathrm{~m}, 1 \mathrm{H}), 6.55(\mathrm{~m}, 1 \mathrm{H}), 4.01$ $(\mathrm{s}, 3 \mathrm{H}), 2.32(\mathrm{~s}, 3 \mathrm{H}) ;{ }^{13} \mathrm{C} \mathrm{NMR}\left(500 \mathrm{MHz}\right.$, acetone- $\left.d_{6}\right) \delta 156.1,136.2,132.9,131.3,131.1,130.3$, $127.7,127.2,124.5,122.2,121.9,112.6,109.9,107.1,56.0,21.4 ; \mathrm{IR}\left(\mathrm{KBr}, \mathrm{cm}^{-1}\right) 3457,1487,1258$ 1232, 779, 745. Anal. Calcd for $\mathrm{C}_{18} \mathrm{H}_{17} \mathrm{NO}: \mathrm{C}, 82.10 ; \mathrm{H}, 6.51$. Found: C, 82.17; H, 6.53.

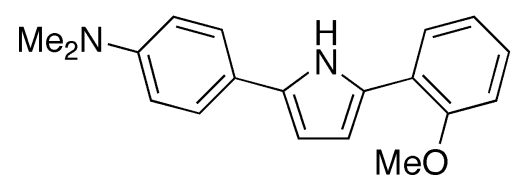

2-(2'-Methoxyphenyl)-5-(4'-dimethylaminophenyl)-1H-pyrrole. General procedure A was followed using 4-bromo- $N, N$-dimethylaniline (0.068 g, $0.34 \mathrm{mmol}), 2$-(2'-methoxyphenyl)pyrrolylsodium (0.100 g, $0.513 \mathrm{mmol}), \mathrm{ZnCl}_{2}(0.070 \mathrm{~g}, 0.513 \mathrm{mmol}), \mathrm{Pd}(\mathrm{OAc})_{2}(3.8 \mathrm{mg}, 0.017 \mathrm{mmol}, 5.0 \mathrm{~mol} \%)$, 2-(di-tertbutylphosphino)biphenyl (5.0 mg, $0.017 \mathrm{mmol}, 5.0 \mathrm{~mol} \%)$, and THF $(1 \mathrm{~mL})$. The reaction was run for 17 hours at $100{ }^{\circ} \mathrm{C}$. Standard workup and isolation, using 70:30 hexanes:ethyl acetate as eluant, afforded the title compound as a red solid, $0.079 \mathrm{~g}(79 \%)$ : $\mathrm{mp} 120-126{ }^{\circ} \mathrm{C}$ (dec.); ${ }^{1} \mathrm{H} \mathrm{NMR}(500 \mathrm{MHz}$, $\left.\mathrm{CDCl}_{3}\right) \delta 9.95($ br s, $1 \mathrm{H}), 7.69(\mathrm{~d}, J=7.3 \mathrm{~Hz}, 1 \mathrm{H}), 7.44(\mathrm{~d}, J=8.7 \mathrm{~Hz}, 2 \mathrm{H}), 7.16(\mathrm{t}, J=7.4 \mathrm{~Hz}, 1 \mathrm{H})$, 6.96-7.03 (m, $2 \mathrm{H}), 6.80(\mathrm{~d}, J=8.7 \mathrm{~Hz}, 2 \mathrm{H}), 6.67(\mathrm{~m}, 1 \mathrm{H}), 6.44(\mathrm{~m}, 1 \mathrm{H}), 4.02(\mathrm{~s}, 3 \mathrm{H}), 3.00$ (br s, 6 $\mathrm{H}) ;{ }^{13} \mathrm{C} \mathrm{NMR}\left(500 \mathrm{MHz}, \mathrm{CDCl}_{3}\right) \delta 154.7,149.4,132.9,129.7,126.5,126.4,125.1,122.1,121.7,121.4$, 113.2, 111.9, 108.0, 105.0, 56.0, 40.9; IR (KBr, $\left.\mathrm{cm}^{-1}\right) 3450,1491,1020,768,741$. Anal. Calcd for $\mathrm{C}_{19} \mathrm{H}_{20} \mathrm{~N}_{2} \mathrm{O}: \mathrm{C}, 78.05 ; \mathrm{H}, 6.89$. Found: C, 77.78; H, 6.89.

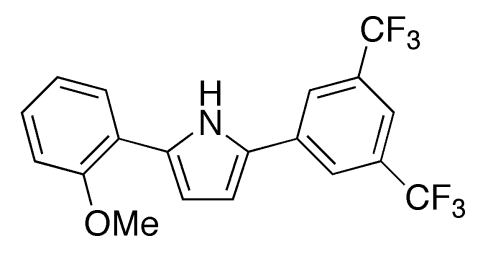

2-[3',5'-Bis(trifluoromethyl)phenyl]-5-(2"-methoxyphenyl)-1H-pyrrole. General procedure A was followed using 3,5-bis(trifluoromethyl)bromobenzene (0.086 mL, $0.50 \mathrm{mmol}), 2$-(2'-methoxyphenyl)pyrrolylsodium (0.196 g, $1.00 \mathrm{mmol}), \mathrm{ZnCl}_{2}(0.138 \mathrm{~g}, 1.00 \mathrm{mmol}), \mathrm{Pd}(\mathrm{OAc})_{2}(5.6 \mathrm{mg}, 0.025 \mathrm{mmol}, 5.0$ 
mol \%), 2-(dicyclohexylphosphino)biphenyl ( $8.8 \mathrm{mg}, 0.025 \mathrm{mmol}, 5.0 \mathrm{~mol} \%$ ), and THF (1 mL). The reaction was run for 54 hours at $100{ }^{\circ} \mathrm{C}$. Standard workup and isolation, using 90:10 hexanes:ethyl acetate as eluant, afforded the title compound as a pale yellow solid, $0.180 \mathrm{~g}(93 \%): \mathrm{mp} 127-128{ }^{\circ} \mathrm{C} ;{ }^{1} \mathrm{H}$ NMR $\left(500 \mathrm{MHz}\right.$, acetone- $\left.d_{6}\right) \delta 10.90(\mathrm{br} \mathrm{s}, 1 \mathrm{H}), 8.29(\mathrm{~s}, 2 \mathrm{H}), 7.75(\mathrm{~s}, 1 \mathrm{H}), 7.71(\mathrm{dd}, J=7.8 \mathrm{~Hz}$ and $1.8 \mathrm{~Hz}, 1 \mathrm{H}), 7.26$ (distorted td, $J=7.8 \mathrm{~Hz}$ and $1.7 \mathrm{~Hz}, 1 \mathrm{H}), 7.11(\mathrm{dd}, J=8.3 \mathrm{~Hz}$ and $1.1 \mathrm{~Hz}, 1 \mathrm{H}$ ), 6.97-7.03 (m, $2 \mathrm{H}), 6.76$ (distorted dd, $J=3.9 \mathrm{~Hz}$ and $2.5 \mathrm{~Hz}, 1 \mathrm{H}), 3.97(\mathrm{~s}, 3 \mathrm{H}) ;{ }^{13} \mathrm{C} \mathrm{NMR}(500 \mathrm{MHz}$, acetone- $\left.d_{6}\right) \delta 206.3,156.7,136.3,133.7,132.6$ (q, $\left.J=32.8 \mathrm{~Hz}\right), 129.9,128.8,128.0,124.7$ (q, $J=272.4$ $\mathrm{Hz}), 124.4,121.8,118.9(\mathrm{~m}, J=4.0 \mathrm{~Hz}), 112.5,111.3,110.8,56.0 ;{ }^{19} \mathrm{~F}$ NMR (300 MHz, acetone- $\left.d_{6}\right) \delta$ -68.1; IR (KBr, $\left.\mathrm{cm}^{-1}\right) 3435,1370,1279,1169,1125,1092,756$. Anal. Calcd for $\mathrm{C}_{19} \mathrm{H}_{13} \mathrm{~F}_{6} \mathrm{NO}: \mathrm{C}$, 59.23; H, 3.40. Found: C, 59.34; H, 3.37.

\section{References}

1. Prepared from anhydrous $\mathrm{ZnCl}_{2}$, weighed in the glovebox, and anhydrous THF; the resulting solution was stored in a resealable Schlenk tube under argon. Solutions of $\mathrm{ZnCl}_{2}$ in THF may also be purchased commercially (e.g. from Aldrich).

2. Rapoport, H.; Look, M. J. Am. Chem. Soc. 1953, 75, 4605.

3. Xu, Z.; Lu, X. J. Org. Chem. 1998, 63, 5031.

4. Guizzardi, B.; Mella, M.; Fagnoni, M.; Albini, A. Tetrahedron 2000, 56, 9383.

5. Ohkura, K.; Seki, K.; Terashima, M.; Kanaoka, Y. Heterocycles 1990, 30, 957. 